
\title{
25 Research Soure \\ Effects of the Pathological E200K Mutation on Human Prion Protein: A Computational screening and Molecular Dynamic approach
}

Fatemeh Rahimi Gharemirshamloo

Ege University

Ranabir Majumder

Indian Institute of Technology Kharagpur

Kourosh Bamdad

Payame Noor University

Fateme Frootan

National Institute of Genetic Engineering \& Biotechnology (NIGEB)

Cemal Un ( $\nabla$ cemaluen@gmail.com )

Ege University

Research Article

Keywords: Pathological E200K Mutation, Prion Protein, Molecular Dynamic

Posted Date: January 6th, 2022

DOI: https://doi.org/10.21203/rs.3.rs-1202156/v1

License: (9) This work is licensed under a Creative Commons Attribution 4.0 International License.

Read Full License 


\section{Abstract}

The Human Prion protein gene (PRNP) is mapped to short arm of chromosome 20 (20pter-12). Prion disease is associated with mutations in the Prion Protein encoding gene sequence. The mutations that occur in the prion protein could be divided into two types based on their influence on pathogenic potential: 1. Mutations that cause disease. 2. Disease-resistance mutations. Earlier studies found that the mutation G127V in the PRNP increases protein stability, whereas the mutation E200K, which has the highest mutation rate in the Prion protein, causes Creutzfeldt-Jakob disease (CJD) in humans and induces protein aggregation. We used a variety of bioinformatic algorithms, including SIFT, PolyPhen, IMutant, PhD-SNP, and SNP\&GO, to predict the association of the E200K mutation with Prion disease. MD simulation is performed and graphs for RMSD, RMSF, Rg, DSSP, PCA, porcupine and FEL are generated to confirm and prove the stability of the wild type and mutant protein structures. The protein is analyzed for aggregation, and the results indicates more fluctuations in the protein structure during the simulation by the E200K mutation, however the G127V mutation makes protein structure stable against aggregation during the simulation.

\section{Introduction}

The accumulation of the pathological form of prion protein in humans' and animals' brains causes prion diseases. Evidence suggests that pathological form of prion protein is created by the transition of natural prion protein (with $42 \%$ a-helix) to abnormal prion protein (with $43 \% \beta$-sheet) that causes the alpha helix deformed beta-sheet structure which is reported in both humans and animals (1-4). These fatal neurodegenerative maladies include symptoms such as rapidly developing dementia, difficulty walking and changes in gait, hallucinations, muscle stiffness, difficulty speaking, fainting, and finally death. Human prion diseases develop in three forms sporadic, genetic, and infectious, which genetic factors account for about $10-15 \%$ of all human prion disorders, such as Creutzfeldt-Jakob disease (CJD), Gerstmann-Straussler-Scheinker (GSS) Syndrome, and Fatal-Familial-Insomnia (FFI) $(5,6)$.

The prion protein gene is located on the short arm of chromosome 20 (20pter-12) which is known as PRNP(7). The primary encoded protein by PRNP has a total of 253 amino acids (two structurally separated segments, including n-terminal and c-terminal), while mature prion protein has only 209 amino acids. The globular domain of prion protein is located at the carboxyl terminus of this protein, globular domain has 3 alpha Helix which includes amino acid sequences 144-154(helix 1), 173-194(helix 2), and 200-228(helix3) respectively, this region also contains two parallel short beta structures, that contain amino acid sequences 128-131 (beta-sheet 1) and 161-164(beta-sheet 2). The disulfide bridge between the cysteine 179 (Cys-179) and cysteine 214 (Cys-214) amino acids bind Helix 2 and Helix 3 together. Two glycosylated areas are located on Asparagine 197 (Asn-197) and Asparagine 181 (Asn181) (8-12). The spatial structure of the globular domain of prion protein is available for testing.

Mutations in the Prion Protein encoding Gene region are responsible for creating the genetic state of the Prion disease. So far, more than 50 pathogen point mutations of the PRNP have been identified in 
connection with a variety of prion diseases (13-15). In terms of their impact on pathogenicity, we can classify the mutations that occur in the prion protein into two categories, which are: - i. Pathogenic mutations, which cause prion diseases in the person carrying the mutation, such as D178V, E200K, etc. (16-19). ii. Disease-preventive mutations in the carrier, such as G127V, M129V, and E219K $(13,20-$ 23). A researcher has been reported E200K nsSNPs dozens of times which demonstrate phenotypic effects in CJD $(24,25)$.

The E200K mutation was first identified by researchers in 1989 and was determined as the dominant Mendelian causative agent of the prion disease in 1991(26). Although many studies have been done on prion diseases, the mechanism of underlying prion diseases has been poorly understood and there is no cure for these diseases. The substitution of glutamate amino acid with lysine amino acid in codon 200 human prion protein (E200K) with the highest mutation rates in this position leads to the development of Creutzfeldt-Jakob disease (CJD) in humans $(14,27)$. New mutations G127V in Papua New Guinea have been identified as completely resistant to Prion disorder, although the mechanism of resistance is unknown (28). It is generally accepted that changes in protein function may be induced by the occurrence of non-synonymous SNP in the coding region, either directly or by conversion in protein structure $(29,30)$.

These days, using computational platforms has become a well-known method for predicting diseaserelated nsSNPs by researchers. In current research, different computational servers were performed for analyze of substitution of glutamate amino acid with lysine in codon 200 PRNP (E200K). A variety of bioinformatic tools including SIFT (31), PolyPhen (32), I-Mutant (33), PhD-SNP (34) and SNP\&GO (35) are used to predict the association of the E200K mutation with disease. In most researches due to prion maladies, molecular dynamics simulations have been used to investigate the cause of prion diseases at the molecular level. In this study, Molecular dynamics simulation approach was performed to find the structural and functional changes of prion protein due to E200K substitution. Gromacs inbuilt tools like g_rms, g_rmsf, g_gyrate, g_covar, g_anaeig, and g_sham were applied for protein trajectories and atomic interaction analysis.

In the current study, one of the most deleterious Prion protein mutations (E200K) is screened with various bioinformatics servers, and compared with prion completely resistances' mutation (G127V) and wild type model of prion protein (WT) by molecular dynamics simulation.

\section{Materials And Methods}

\section{Disease-associated nsSNP prediction}

The Sorting Intolerant from Tolerant (SIFT) algorithm was used to evaluate an amino acid substitution affects protein function based on sequence homology and the physical properties of amino acids. In SIFT server prediction score less than 0.05 is considered as deleterious for amino acid substitution. Polymorphism Phenotyping (PolyPhen) is a server that predicts the effect of an amino acid substitution on the structure and function of a human protein, damaging nSSNP predicted by a PSIC score of more than 0.05. I-Mutant server is a technique with the SVM (Support Vector Machines) method and the basis 
of this tool is protein stability. For a score less than zero, the nsSNP is considered harmful. I-Mutant predicts free energy change (DDG) that was based on the difference between unfolding Gibbs free energy change of mutant and wild type protein. In addition, we used SNPs\&GO and PhD-SNP to predict the association of the mutation with prion disease.

\section{Data-set}

In the first, the information of human PRNP nsSNP (E200K) as deleterious nsSNP was collected from published articles $(14,36-38)$. Then the amino acid sequence of Human Prion protein was extracted from the Uniprot Database (Uniprot ID: P04156). And The crystallographic structure of the Human Prion Protein (PRNP) was obtained from Brookhaven Protein Data Bank (PDB ID: 1HJM) (12). In this research, we used SIFT, Polyphen-2, I-Mutant 3.0, SNPs\&GO, and PhD-SNP to analyze E200K, which reveals the impact of this mutation on prion illness. After that SPDB viewer was used to search for missing atoms and performed energy minimization. The modifications of the PDB structure were achieved by Accelrys Discovery Studio 4.1(39) and Pymol (40). Finally, energetically optimization and MD simulations were performed by GROMACS 5 (41).

\section{Molecular dynamics simulation}

Molecular dynamics simulation for all structures was done by Gromacs 5 (41) package on running Linux Ubuntu 16.04 operating system. We used force field GROMOS96 43a1 (42) for simulation. Wild type and mutant proteins were solvated in a cubic box with a dimension of $52.0 \mathrm{~A}^{\circ}$ including single-point-charge (SPC) water. To create the electrically neutral simulation system, the structures were neutralized by adding $\mathrm{Na}^{+}$and $\mathrm{Cl}^{-}$ions at physiological pH. Energy minimization was performed for 1000 steps by the steepest descent methods. Then molecular dynamic simulation was carried out in three steps containing NVT-MD (constant number of particles [N], volume [V], and temperature [T]), NPT-MD (constant number of particles [N], pressure [P], and temperature $[\mathrm{T}]$ ) for 1000 picoseconds (ps) at $300 \mathrm{~K}$ and the final simulation production were carried out at $300 \mathrm{k}$ for 300 ns for wild type protein, E200K and G127V models. We then calculated the comparative analysis of structural deviations in the wild-type and mutant structures.

\section{Results}

\section{Prediction of association between E200K mutation and Prion disease}

In this study bioinformatics servers such as SIFT, PolyPhen, I-Mutant, SNP\&GO, and PhD-SNP have been used to understand the mutation (E200K) association with disease. In the first step, the result of the analysis of the E200K mutation by SIFT server $(p=0.01)$ was predicted to be deleterious. The result of PolyPhen $(P S I C=1)$ showed its effect on protein structure and function. Also, the output of the I-Mutant server $(D G G=-0.28)$ represented the effect of the E200K mutation on the protein structure instability (Table 1). Further both servers SNP\&GO and PhD-SNP results revealed the association of E200K mutation to prion disease (Table 2). 
Table 1: E200K SNP analyzed by three bioinformatics servers SIFT, PolyPhen2 and I-Mutant3.0
I-Mutant
PolyPhen2
SIFT
Mutation SNPID
3.0

Stability

Prediction

Prediction Score

DDG

PSIC

PSIC Prediction Score

$\begin{array}{llllllll}\text { Decrease } & -0.28 & \begin{array}{l}\text { Probably } \\ \text { damaging }\end{array} & 1 & \text { Deleterious } & 0.01 & \text { E200K } & \text { rs28933385 }\end{array}$

Table 2: Prediction of disease association of mutation E200K by 2 computational methods SNP\&GO and PhD-SNP

\begin{tabular}{llllll} 
& PhD SNP & & SNP\&GO & Mutation & SNP ID \\
\hline RI & EFFECT & RI & EFFECT & & \\
\hline & & & & & \\
\hline 6 & Disease & 8 & Disease & E200K & rs28933385
\end{tabular}

\section{Protein dataset}

The structure of the wild-type PRNP (WT) was obtained from the protein databank (PDB: 1HJM). To better understand the effects of mutation on protein dynamics, we classified prion structures into three categories based on secondary structure, three a-helix ( $\mathrm{H} 1, \mathrm{H} 2$, and $\mathrm{H} 3)$, two beta sheets ( $\mathrm{S} 1$ and $\mathrm{S} 2)$, and a disulfide bridge (DB) (Figue 1A). The co-crystallized water molecules were removed. Using Swiss PDB Viewer, Discovery Studio, and Pymol, point mutations are induced at positions 127 and 200 of the wild type human prion protein, with Glycine(G) at location 127 replaced by Valine(V) and Glutamate(E) at position 200 replaced by Lysine(K) (Figure 1B).

\section{Molecular Dynamics Simulation}

We performed the molecular dynamics simulation of the wild type (WT) and mutant structures containing E200K and G127V over for a period of 300ns to understand structural dynamics and stability effect of mutant models on prion protein. The five factors, containing, tolerance index, PSIC score, DDG value, subPSEC score, and disease-association study, confirm the conformational changes in Prion protein residues due to the mutation (E200K), which can change the functional behavior of protein molecules. The importance of the resistant effect of G127V mutation to prion disease and the results obtained in the above analysis on E200K mutation, further motivated us to monitor the dynamic behavior of wild, and mutant types (E200k, and G127V) variants of prion proteins. Then we performed root mean square deviation (RMSD), secondary structure (DSSP), root mean square fluctuation (RMSF), radius of gyration $(\mathrm{Rg})$, porcupine plots, and Gibbs' free energy landscape (FEL) analysis of all the structures (wild type, E200K and G127V) in the prion protein. 
Root-mean-square deviation (RMSD) values were calculated for 3 model structures over 300 ns (WT, G127V, and E200K) (Figure 2). The backbone RMSD value ranged from 0.15 to $0.4 \mathrm{~nm}$ for all trajectories. During the first $100 \mathrm{~ns}$, the WT model indicates an increasing trend from 0.15 to $0.38 \mathrm{~nm}$. Suddenly, the RMSD value of WT during the $100-150 \mathrm{~ns}$ dropped to $0.16 \mathrm{~nm}$ and till the end of the simulation, the WT model showed the lower RMSD measure as compared to G127V and E200K structures respectively. G127V model shows an increasing trend from 0.15 to $0.35 \mathrm{~nm}$ during the first $20 \mathrm{~ns}$. After that, till $75 \mathrm{~ns}$ the measure of RMSD decreased slightly to $0.16 \mathrm{~nm}$, and then the amount of G127V grew slowly up to $170 \mathrm{~ns}$ and reached $0.35 \mathrm{~nm}$ and till to end of the simulation RMSD remained stable in the case of the G127V model. E200K model demonstrates a rising trend from $0 \mathrm{~ns}$ to $25 \mathrm{~ns}$ about 0.2 to $0.38 \mathrm{~nm}$, however after that till $75 \mathrm{~ns}$ RMSD decreases to almost $0.22 \mathrm{~nm}$. Then RMSD increases to $4 \mathrm{~nm}$ at 100ns dramatically. According to the RMSD plot, the E200K model attains the maximum deviation till the end of the simulation compared to wild type and G127V structure. RMSD plot demonstrates major stability loss in 3 parts of simulation time, such as 40-50, 90-110, and 140-175 ns for the E200K model. The information's obtained from the RMSD plot illustrates that on an average amount of instability E200K > WT > G127V respectively.

\section{RMSF}

Figure 3(A) root-mean-square fluctuations (RMSFs) of native and mutant (E200K and G127V) structures during the simulations. The highest RMSFs scores were observed in the E200K structure, due to E200k mutation, two peaks remarked in the $\beta 1$ and end of helix 2, which localized around the residues 132-134 and 191-196(LYS194) respectively. An unusual peak in the model E200K has been observed in the $\beta 1$ region with a value of approximately $0.43 \mathrm{~nm}$, which is correlated with the MET134 amino acid. The highest fluctuation is remarked in the C-terminus of Helix 2, with a maximum value of approximately 0.56 $\mathrm{nm}$ which is correlated with the LYS194 amino acid. G127V as a protective mutation demonstrates less root-mean-square fluctuation than 2 other structures (E200K, WT) at the end of Helix 2. The central parts of Helix 2 and Helix 3 show the lowest RMSFs score. As shown in Figure 3(B), the order of mentioned RMSF instability for all structures is E200K $>$ WT $>$ G127V respectively.

\section{Secondary Structural Analysis (DSSP)}

Secondary structural elements as a function of simulation are shown in Figure 4. It has been observed that the wild type and G127V models follow the same pattern during the simulation with less fluctuation. However, the G127V model is more stable than WT in the period of simulation time. On the other hand, the most changes in the secondary structure of the protein were related to the C-terminus of Helix 2 and B1-strand in the E200K model during the simulation. Although results obtained from secondary structure have depicted fluctuation at the end of helix 2 for all 3 models (WT, G127V, E200K), the E200K has shown extensive conversion from helix to turn, bend, b-bridge, and b-sheet that support the RMSF pattern in

Figure 3. The significant variation of the secondary structure is related to the C-terminus of Helix-2 at 50, 100, 150, 220 ns, with variations observed as B-Sheet and B-Bridge for E200K mutant result, which the 
peak of changes is related to $100 \mathrm{~ns}$ in the c-terminus of helix2 that conversion to B-sheet is seen. In the C-terminus of Helix-2 due to E200K, during the simulation, variations in the form of the Bend, Turn, Bbridge and B-sheet are always seen, which is related to amino acids 189-197. During the simulation, B1 is extended. Changes at the C-terminus Helix 3 are visible as Turn and Bend in all 3 models (WT, G127V, E200K). The order of secondary structure deviation is E200K $>W T>G 127 V$ respectively.

\section{Radius of gyration $(\mathbf{R g})$}

Figure 5 shows the radius of gyration of the protein $(\mathrm{Rg})$ plot obtained by the g_gyrate tool of the Gromacs package. On average, the Rg value for all structures ranged between 1.37 to $1.52 \mathrm{~nm}$. The radius of gyration of native protein primarily decreased from 1.5 to 1.4 until $150 \mathrm{~ns}$ but the Rg value increased the last 100 nanoseconds of the simulation to approximately $1.5 \mathrm{~nm}$. Rg plot for E200K structure represents a significant decrease from 1.52 up to $1.37 \mathrm{~nm}$ from the $25 \mathrm{~ns}$ until $125 \mathrm{~ns}$ of simulation, then the Rg value increases up to $152 \mathrm{~ns}$ at $170 \mathrm{~nm}$. In the G127V structure, the first $100 \mathrm{~ns}$ $\mathrm{Rg}$ value increased up to $1.51 \mathrm{~nm}$ and this value is decreased for this model to $1.38 \mathrm{~nm}$ the last $150 \mathrm{~ns}$ of simulation.

\section{Radius of gyration (Rg)}

Figure 5 shows the radius of gyration of the protein $(\mathrm{Rg})$ plot obtained by the g_gyrate tool of the Gromacs package. On average, the Rg value for all structures ranged between 1.37 to $1.52 \mathrm{~nm}$. The radius of gyration of native protein primarily decreased from 1.5 to 1.4 until $150 \mathrm{~ns}$ but the Rg value increased the last 100 nanoseconds of the simulation to approximately $1.5 \mathrm{~nm}$. Rg plot for E200K structure represents a significant decrease from 1.52 up to $1.37 \mathrm{~nm}$ from the $25 \mathrm{~ns}$ until $125 \mathrm{~ns}$ of simulation, then the Rg value increases up to $152 \mathrm{~ns}$ at $170 \mathrm{~nm}$. In the G127V structure, the first $100 \mathrm{~ns}$ Rg value increased up to $1.51 \mathrm{~nm}$ and this value is decreased for this model to $1.38 \mathrm{~nm}$ the last $150 \mathrm{~ns}$ of simulation.

\section{Porcupine analysis}

The motion's direction and magnitude, corresponding to the first two principal components (PC1 and PC2) constructed from 300ns molecular dynamics simulation are depicted by the red conic arrows in Porcupine plots in Figure 7. Overall, the E200K model shows more motion compared to the WT and G127V models, furthermore porcupine plots indicate that G127V has the least movement. In this analysis, most fluctuations in motion are related to the beginning of the globular domain, the end of B-sheet1, helix $2 \mathrm{c}$-terminal, and the part between helix 2 and helix 3 and the end of helix 3 in model E200K. The order of the relative amplitude of motion of the $\mathrm{Ca}$ atoms in three models is E200K $>W T>\mathrm{G} 127 \mathrm{~V}$ respectively.

\section{Gibbs' Free energy landscape}

The free-energy landscape (FEL) framework is one way of describing protein folding, which is still an open subject in science. Figure 8 represents the EFL plot for wild type (WT), E200K, and G127V models. FEL can show the global minima energy conformation of a biostructure (protein). If biostructure has low 
stability, it can attain multiple minimum energy clusters. On other hand, a strong and stable structure conformation can achieve almost a single conformation energy clusture(43). GROMACS inbuilt scripts (g_covar, g_anaeig, and g_sham) were used to calculate the first two principal components (PC1 and PC2) as reaction coordinates, resulting in FEL for three models. The basic function of g_sham is to plot Gibbs free energy landscapes by Bolzmann inverting multi-dimensional histograms with PC1 and PC2 as input. In the FEL plots, Dark violet/blue regions reflect the energy minima and energetically favoured structural conformation and red/yellow regions indicate the unfavourable structural conformation. The shallow and narrow energy basin indicates the low stability of structural conformation. According to the FEL plots in Figure. 8, the G127V showed more stability compared to the WT and E200K models.

\section{Discussion And Conclusion}

Single-nucleotide polymorphisms (SNPs) are the cause of more genetic diversity in humans. These polymorphisms appear in the coding region of the protein in two forms (types), synonymous single nucleotide polymorphisms and non-synonymous single nucleotide polymorphisms (nsSNPs). Among these single-nucleotide polymorphisms (SNPs), non-synonymous single-nucleotide polymorphisms (nsSNPs) cause amino acid residue change in the protein sequence $(44,45)$. Which can cause changes in protein function due to changes in protein structure and folding (46-48). In silico methods are the most common approach to gathering information about the etiology of prion diseases by researchers (4952). Therefore, due to the importance of the role of non-synonymous single nucleotide polymorphisms (nsSNPs), we used a variety of prediction methods and algorithms to analyze the E200K mutation, which destabilizes the Prion protein. E200K mutation was predicted deleterious by using SIFT, Polyphen and I-Mutant tools, with SIFT score $=0.01, \mathrm{PSIC}=1.000$, and DDG $=-0.28$ (table 1 ). Moreover, this nsSNP was predicted disease-associated by using PhD-SNP and SNP\&GO servers (Table 2). Molecular dynamic simulation, A powerful computational method, performed for the Prion disease-associated nsSNP (E200K) model, G127V model (as resistant nsSNP to prion disease), and wild type (WT) models of prion protein to examine the structural dynamics and the stability of E200K and G127V models as compared to wild type structure.

The RMSD computed for all $\mathrm{Ca}$ atoms from starting structures to describe the convergence of the protein system. RMSD values were plotted for E200K, G127V, and native structure (Figure 2). As represented by Yun et al. [2011], a larger RMSD is related to less stability (53), The highest fluctuation in the RMSD plot is related to the E200K model is visible with a maximum value of approximately $0.4 \mathrm{~nm}$ at almost $100 \mathrm{~ns}$. And the lowest value is related to the G127V structure with a minimum value of approximately $0.15 \mathrm{~nm}$ at about 75 and 230 ns. The RMSF of Ca atoms between the residues for three structures (E200K, G127V, and wild type) were calculated to detect the effect of mutation on the dynamic behavior of residues (Figure 3). Considering that the maximum value of RMSFs occurs at the end of B-sheet 1, helix 2, and Helix 3. So, it can be suggested that these regions of the protein have a key role in conformational rearrangements. G127V as a protective mutation demonstrates less root-mean-square fluctuation than other structures (E200K, and WT) at the end of Helix2, which can suggest that this stability is a result of the protective role of this mutation. However, the lowest RMSFs score is related to the central parts of 
Helix 2 and Helix 3, in which there is a single disulfide bridge between these two helixes (Cys179 Cys214). therefore, it may be supposed that this connection makes the protein more stable in mentioned areas $(9,54-56)$.

Maps of secondary structure changes obtained from MD simulations are depicted in Figure 4. The most changes in the secondary structure of the protein are related to the C-terminus of Helix 2 and B1-strand in the E200K model during the $300 \mathrm{~ns}$ simulation. The E200K model has shown extensive conversion from helix to turn, bend, b-bridge, and b-sheet. As a result, we have observed higher RMSF values in E200K model residues (Figure 3). Therefore, the loss of stability in the E200K mutant structure is suggested by the consequence of protein aggregation in the E200K mutant model. The graph of Radius of gyration for alpha-carbon of the protein versus time at $300 \mathrm{k}$ is depicted in Figure 5. During the whole MD simulation, the $\mathrm{Rg}$ value of the G127V structure is found to drop. In comparison to the G127V model, the E200K and WT structures show more flexibility. This confirmed our theory far more. According to the average Rg graph, the G127V structure was found stable than E200K and the native model. The outcomes of the PCA, porcupine, and FEL plots indicated that the E200K model had a destabilizing impact as compared to the WT and G127V models. According to the porcupine plot, the end of beta-sheet 1, helix 2, and helix 3, and the portion between helix 2 and helix 3 exhibit more fluctuation. Furthermore, porcupine plots reveal that G127V has the least movement and the EFL plot indicates higher stability for the G127V structure in contrast to the WT and E200K models.

The molecular dynamics simulation study on a normal and mutated E200K protein model demonstrates that the E200K mutation caused a change in the secondary structure of the protein. This study's results indicate that the end of B-sheet 1, Helix 2, Helix 3, and the turning region between Helix 2 and Helix 3 can be proposed as a site for initiating protein alterations to $\beta$-sheet and aggregation to amyloids. According to the study on prion protein resistance by $\mathrm{G} 127 \mathrm{~V}$ mutation against prion diseases $(28,57)$ and the results obtained from the MD simulation outputs, it can be interpreted that The G127V mutation prevents structural changes and fluctuations in the suggested amyloid initiation region and makes these regions more stable against fluctuations and structural rearrangement $(12,58-61)$. We have analyzed one of the most deleterious Prion protein nsSNP (E200K) with a various number of bioinformatics servers and also compared our result with some experimentally reported phenotypic effects of the E200K nsSNPs. On the other hand, we have performed a molecular dynamics simulation of the E200K structure, G127V structure, and native protein structure. Due to information obtained from the server's screen and molecular dynamic simulation analyses, it can be understood that some mutation such as E200K in the sequence of protein has a loss of stability effect and make aggregation in protein folding and some rare mutation like G127V make protein more stable against disease.

Overall, according to the results obtained from the prediction of servers that the E200K mutation occurs as a deleterious mutation in the prion protein structure. The simulation results (RMSD, RMSF, RG, PCA, Porcupine, FEL, and DSSP) shows more fluctuations in the protein structure during the simulation by the E200K mutation at the end of B-sheet 1, Helix 2, Helix 3, and the turning region between Helix 2-3, the G127V mutation makes protein structure stable against aggregation during the simulation. we propose to 
perform more experimental and computational research on both deleterious and resistant mutations to find the mechanism of change in protein structure and folding by these two mutant groups (deleterious and resistant).

\section{References}

1. Prusiner SB. Prions. Proceedings of the National Academy of Sciences of the United States of America. 1998;95(23):13363-83.

2. Prusiner SB, Scott MR, DeArmond SJ, Cohen FE. Prion protein biology. Cell. 1998;93(3):337-48.

3. Kaneko K, Zulianello L, Scott M, Cooper CM, Wallace AC, James TL, et al. Evidence for protein X binding to a discontinuous epitope on the cellular prion protein during scrapie prion propagation. Proceedings of the National Academy of Sciences of the United States of America. 1997;94(19):1006974.

4. Pan KM, Baldwin M, Nguyen J, Gasset M, Serban A, Groth D, et al. Conversion of alpha-helices into beta-sheets features in the formation of the scrapie prion proteins. Proceedings of the National Academy of Sciences of the United States of America. 1993;90(23):10962-6.

5. DeArmond SJ, Prusiner SB. Etiology and pathogenesis of prion diseases. The American journal of pathology. 1995;146(4):785-811.

6. Wadsworth JD, Hill AF, Beck JA, Collinge J. Molecular and clinical classification of human prion disease. British medical bulletin. 2003;66:241-54.

7. Puckett C, Concannon P, Casey C, Hood L. Genomic structure of the human prion protein gene. American journal of human genetics. 1991;49(2):320-9.

8. Viles JH, Cohen FE, Prusiner SB, Goodin DB, Wright PE, Dyson HJ. Copper binding to the prion protein: structural implications of four identical cooperative binding sites. Proceedings of the National Academy of Sciences of the United States of America. 1999;96(5):2042-7.

9. Zahn R, Liu A, Lührs T, Riek R, von Schroetter C, López García F, et al. NMR solution structure of the human prion protein. Proceedings of the National Academy of Sciences of the United States of America. 2000;97(1):145-50.

10. DeMarco ML, Silveira J, Caughey B, Daggett V. Structural properties of prion protein protofibrils and fibrils: an experimental assessment of atomic models. Biochemistry. 2006;45(51):15573-82.

11. Cobb NJ, Sönnichsen FD, McHaourab H, Surewicz WK. Molecular architecture of human prion protein amyloid: a parallel, in-register beta-structure. Proceedings of the National Academy of Sciences of the United States of America. 2007;104(48):18946-51. 
12. Calzolai L, Zahn R. Influence of pH on NMR structure and stability of the human prion protein globular domain. Journal of Biological Chemistry. 2003;278(37):35592-6.

13. Zheng Z, Zhang M, Wang Y, Ma R, Guo C, Feng L, et al. Structural basis for the complete resistance of the human prion protein mutant G127V to prion disease. Scientific reports. 2018;8(1):13211.

14. Friedman-Levi Y, Meiner Z, Canello T, Frid K, Kovacs GG, Budka H, et al. Fatal prion disease in a mouse model of genetic E200K Creutzfeldt-Jakob disease. PLoS pathogens. 2011;7(11):e1002350.

15. Synofzik M, Bauer P, Schöls L. Prion mutation D178N with highly variable disease onset and phenotype. Journal of Neurology, Neurosurgery \& Psychiatry. 2009;80(3):345-6.

16. Shamsir MS, Dalby AR. One gene, two diseases and three conformations: molecular dynamics simulations of mutants of human prion protein at room temperature and elevated temperatures. Proteins: Structure, Function, and Bioinformatics. 2005;59(2):275-90.

17. Capellari S, Parchi P, Russo CM, Sanford J, Sy M-S, Gambetti P, et al. Effect of the E200K mutation on prion protein metabolism: comparative study of a cell model and human brain. The American journal of pathology. 2000;157(2):613-22.

18. Zhang Y, Swietnicki W, Zagorski MG, Surewicz WK, Sönnichsen FD. Solution structure of the E200K variant of human prion protein: Implications for the mechanism of pathogenesis in familial prion diseases. Journal of Biological Chemistry. 2000;275(43):33650-4.

19. Gharemirshamlu FR, Bamdad K, Naeimi S. Atomic insight into prion disorder: An intricate detail gained by $0.5 \mu$ s molecular dynamics simulation of preventive G127V and deleterious D178V mutation in prion protein. Journal of cellular biochemistry. 2019;120(8):14156-64.

20. Asante EA, Smidak M, Grimshaw A, Houghton R, Tomlinson A, Jeelani A, et al. A naturally occurring variant of the human prion protein completely prevents prion disease. Nature. 2015;522(7557):478-81.

21. Sabareesan AT, Udgaonkar JB. The G126V mutation in the mouse prion protein hinders nucleation-dependent fibril formation by slowing initial fibril growth and by increasing the critical concentration. Biochemistry. 2017;56(44):5931-42.

22. Bagyinszky E, Van Giau V, Youn YC, An SSA, Kim S. Characterization of mutations in PRNP (prion) gene and their possible roles in neurodegenerative diseases. Neuropsychiatric disease and treatment. 2018;14:2067.

23. Hizume M, Kobayashi A, Teruya K, Ohashi H, Ironside JW, Mohri S, et al. Human prion protein (PrP) $219 \mathrm{~K}$ is converted to PrPSc but shows heterozygous inhibition in variant Creutzfeldt-Jakob disease infection. Journal of Biological Chemistry. 2009;284(6):3603-9. 
24. Rosenmann H, Talmor G, Halimi M, Yanai A, Gabizon R, Meiner Z. Prion protein with an E200K mutation displays properties similar to those of the cellular isoform $\operatorname{PrP}(C)$. Journal of neurochemistry. $2001 ; 76(6): 1654-62$.

25. Paciotti R, Storchi L, Marrone A. An insight of early PrP-E200K aggregation by combined molecular dynamics/fragment molecular orbital approaches. Proteins. 2019;87(1):51-61.

26. Minikel EV, Zerr I, Collins SJ, Ponto C, Boyd A, Klug G, et al. Ascertainment bias causes false signal of anticipation in genetic prion disease. American journal of human genetics. 2014;95(4):371-82.

27. Pocchiari M, Poleggi A, Puopolo M, D’Alessandro M, Tiple D, Ladogana A. Age at Death of Creutzfeldt-Jakob disease in subsequent family generation carrying the E200K mutation of the prion protein gene. PLoS One. 2013;8(4):e60376.

28. Asante EA, Smidak M, Grimshaw A, Houghton R, Tomlinson A, Jeelani A, et al. A naturally occurring variant of the human prion protein completely prevents prion disease. Nature. 2015;522(7557):478-81.

29. Vinay Kumar C, Kumar KM, Swetha R, Ramaiah S, Anbarasu A. Protein aggregation due to nsSNP resulting in P56S VABP protein is associated with amyotrophic lateral sclerosis. Journal of theoretical biology. 2014;354:72-80.

30. Kamaraj B, Rajendran V, Sethumadhavan R, Kumar CV, Purohit R. Mutational analysis of FUS gene and its structural and functional role in amyotrophic lateral sclerosis 6 . Journal of biomolecular structure \& dynamics. 2015;33(4):834-44.

31. Kumar P, Henikoff S, Ng PC. Predicting the effects of coding non-synonymous variants on protein function using the SIFT algorithm. Nature protocols. 2009;4(7):1073-81.

32. Adzhubei IA, Schmidt S, Peshkin L, Ramensky VE, Gerasimova A, Bork P, et al. A method and server for predicting damaging missense mutations. Nature methods. 2010;7(4):248-9.

33. Capriotti E, Fariselli P, Rossi I, Casadio R. A three-state prediction of single point mutations on protein stability changes. BMC bioinformatics. 2008;9 Suppl 2(Suppl 2):S6.

34. Capriotti E, Calabrese R, Casadio R. Predicting the insurgence of human genetic diseases associated to single point protein mutations with support vector machines and evolutionary information. Bioinformatics (Oxford, England). 2006;22(22):2729-34.

35. Calabrese R, Capriotti E, Fariselli P, Martelli PL, Casadio R. Functional annotations improve the predictive score of human disease-related mutations in proteins. Human mutation. 2009;30(8):1237-44.

36. Minikel EV, Zerr I, Collins SJ, Ponto C, Boyd A, Klug G, et al. Ascertainment bias causes false signal of anticipation in genetic prion disease. The American Journal of Human Genetics. 
2014;95(4):371-82.

37. Kosorinova D, Belay G, Zakova D, Stelzer M, Mitrova E. Genetic Risk Factors of Creutzfeldt-Jakob Disease in the Population of Newborns in Slovakia. Pathogens. 2021;10(4):435.

38. Jani V, Sonavane U, Joshi R. Detecting early stage structural changes in wild type, pathogenic and non-pathogenic prion variants using Markov state model. RSC advances. 2019;9(25):14567-79.

39. Studio D. Accelrys Inc. San Diego, CA. 2013.

40. DeLano WL. The PyMOL molecular graphics system. http://www pymol org. 2002.

41. Abraham MJ, Murtola T, Schulz R, Páll S, Smith JC, Hess B, et al. GROMACS: High performance molecular simulations through multi-level parallelism from laptops to supercomputers. SoftwareX. 2015;1:19-25.

42. Schuler LD, Daura X, Van Gunsteren WF. An improved GROMOS96 force field for aliphatic hydrocarbons in the condensed phase. Journal of computational chemistry. 2001;22(11):1205-18.

43. Majumder R, Das CK, Banerjee I, Jena BC, Mandal A, Das P, et al. Screening of the Prime bioactive compounds from Aloe vera as potential anti-proliferative agents targeting DNA. Computers in Biology and Medicine. 2021:105052.

44. Cargill M, Altshuler D, Ireland J, Sklar P, Ardlie K, Patil N, et al. Characterization of singlenucleotide polymorphisms in coding regions of human genes. Nature genetics. 1999;22(3):231-8.

45. Collins FS, Brooks LD, Chakravarti A. A DNA polymorphism discovery resource for research on human genetic variation. Genome research. 1998;8(12):1229-31.

46. Rajasekaran R, Sudandiradoss C, Doss CGP, Sethumadhavan R. Identification and in silico analysis of functional SNPs of the BRCA1 gene. Genomics. 2007;90(4):447-52.

47. Yazar M, Özbek P. In Silico Tools and Approaches for the Prediction of Functional and Structural Effects of Single-Nucleotide Polymorphisms on Proteins: An Expert Review. OMICS: A Journal of Integrative Biology. 2021;25(1):23-37.

48. Cheng TM, Lu Y-E, Vendruscolo M, Lio' P, Blundell TL. Prediction by graph theoretic measures of structural effects in proteins arising from non-synonymous single nucleotide polymorphisms. PLoS computational biology. 2008;4(7):e1000135.

49. Doss CGP, Rajith B, Rajasekaran R, Srajan J, Nagasundaram N, Debajyoti C. In silico analysis of prion protein mutants: A comparative study by molecular dynamics approach. Cell biochemistry and biophysics. 2013;67(3):1307-18. 
50. Sekijima M, Motono C, Yamasaki S, Kaneko K, Akiyama Y. Molecular dynamics simulation of dimeric and monomeric forms of human prion protein: insight into dynamics and properties. Biophysical journal. 2003;85(2):1176-85.

51. Jomah AF, Parvizpour S, Razmara J, Shamsir MS. The structural transformation comparison of the human Prion protein mutants V176G, E196A, and I215V by using molecular dynamics simulation. 2021.

52. Halder P, Mitra P. Human prion protein: exploring the thermodynamic stability and structural dynamics of its pathogenic mutants. Journal of Biomolecular Structure and Dynamics. 2021:1-17.

53. Yun S, Guy HR. Stability tests on known and misfolded structures with discrete and all atom molecular dynamics simulations. Journal of Molecular Graphics and Modelling. 2011;29(5):663-75.

54. Li Y, Yan J, Zhang X, Huang K. Disulfide bonds in amyloidogenesis diseases related proteins. Proteins: Structure, Function, and Bioinformatics. 2013;81(11):1862-73.

55. Benetti F, Legname G. New insights into structural determinants of prion protein folding and stability. Prion. 2015;9(2):119-24.

56. Shin JY, Shin JI, Kim JS, Yang YS, Shin Y-K, Kim KK, et al. Disulfide bond as a structural determinant of prion protein membrane insertion. Molecules and cells. 2009;27(6):673-80.

57. Tang Y, Wei G. Dissecting the Structural Mechanism of a Naturally Occuring Variant of the Prion Protein in Preventing Prion Disease. Biophysical Journal. 2018;114(3):234a.

58. Sanz-Hernández M, Barritt JD, Sobek J, Hornemann S, Aguzzi A, De Simone A. Mechanism of misfolding of the human prion protein revealed by a pathological mutation. Proceedings of the National Academy of Sciences. 2021;118(12).

59. Taguchi Y, Mistica AM, Kitamoto T, Schätzl HM. Critical significance of the region between Helix 1 and 2 for efficient dominant-negative inhibition by conversion-incompetent prion protein. PLoS pathogens. 2013;9(6):e1003466.

60. Singh J, Kumar H, Sabareesan AT, Udgaonkar JB. Rational stabilization of helix 2 of the prion protein prevents its misfolding and oligomerization. Journal of the American Chemical Society. 2014;136(48):16704-7.

61. Tang Y, Yao Y, Wei G. Structural and dynamical mechanisms of a naturally occurring variant of the human prion protein in preventing prion conversion. Chinese Physics B. 2020;29(10):108710.

\section{Figures}


A

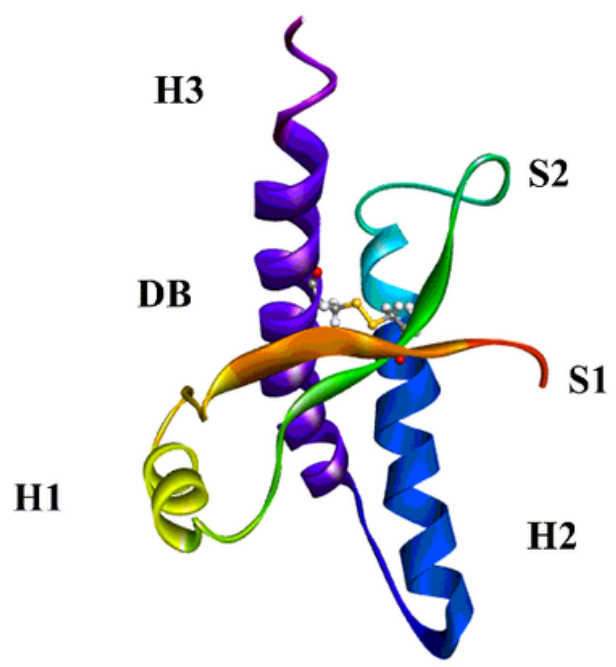

B

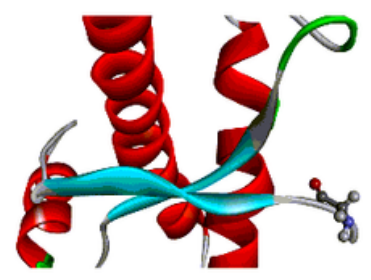

G127

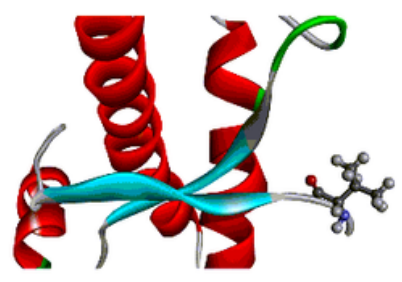

G127V

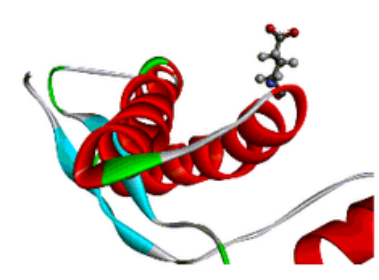

E200

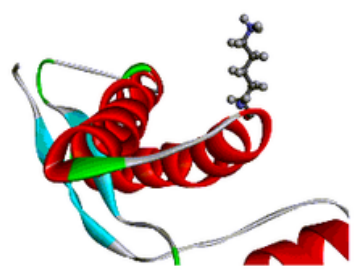

E200K

Figure 1

(A) Secondary structural architecture of the Prion Protein globular domain, revealing two beta sheets (S1 and S2), three helical structures ( $\mathrm{H} 1, \mathrm{H} 2$, and $\mathrm{H} 3$ ), and disulfide bridge (DB). (B) Stick-ball structure for wild type(G127 and E200) and mutant structures(G127V, E200K). 


\section{RMSD}

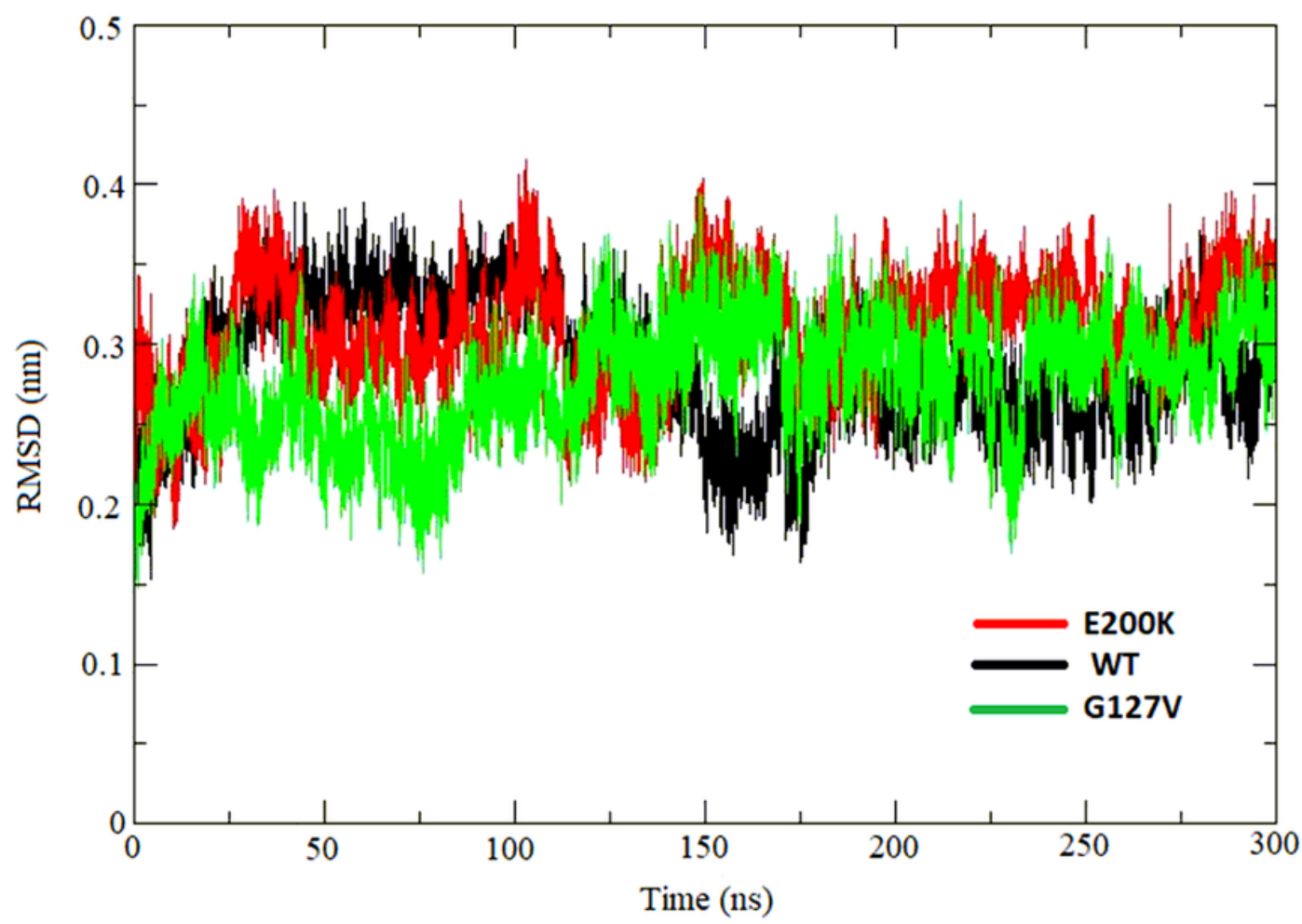

Figure 2

Root-mean square (RMSDs) for native (WT) and mutant Prion protein structures (G127V, E200K).

RMS fluctuation

$\mathbf{A}$

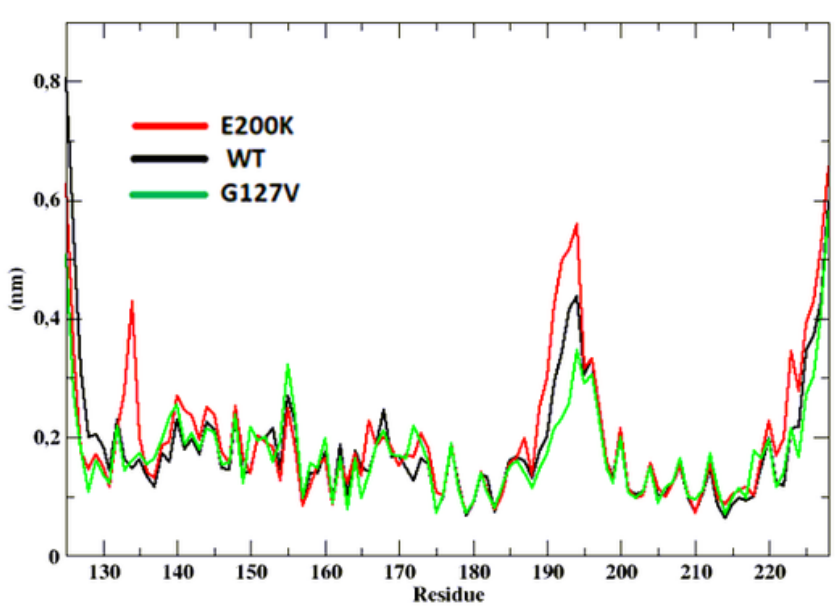

B

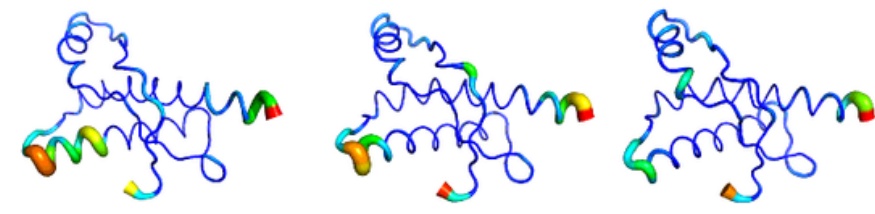

WT

E200K

G127V 
Figure 3

(A) Root-mean-square fluctuations (RMSFs) of Ca atoms of native (WT) and mutant structures (G127V, E200K). (B) Calculated RMSF values converted to B-factor values, showing the regions of flexible and rigid nature for WT (wild type), E200K, and G127V models.


\section{Figure 4}

The secondary structural elements of Prion protein for native (WT) and mutant structures (G127V, E200K). 




Figure 5

Radius of gyration of Ca atoms of native (WT), resistant SNP (G127V), and mutation (E200K). 
A Eigenvalues of the covariance matrix

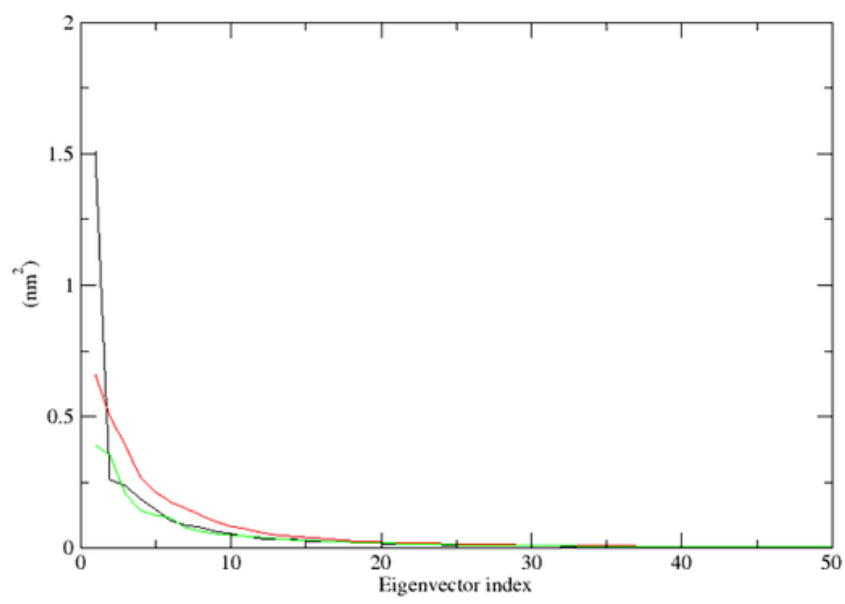

B

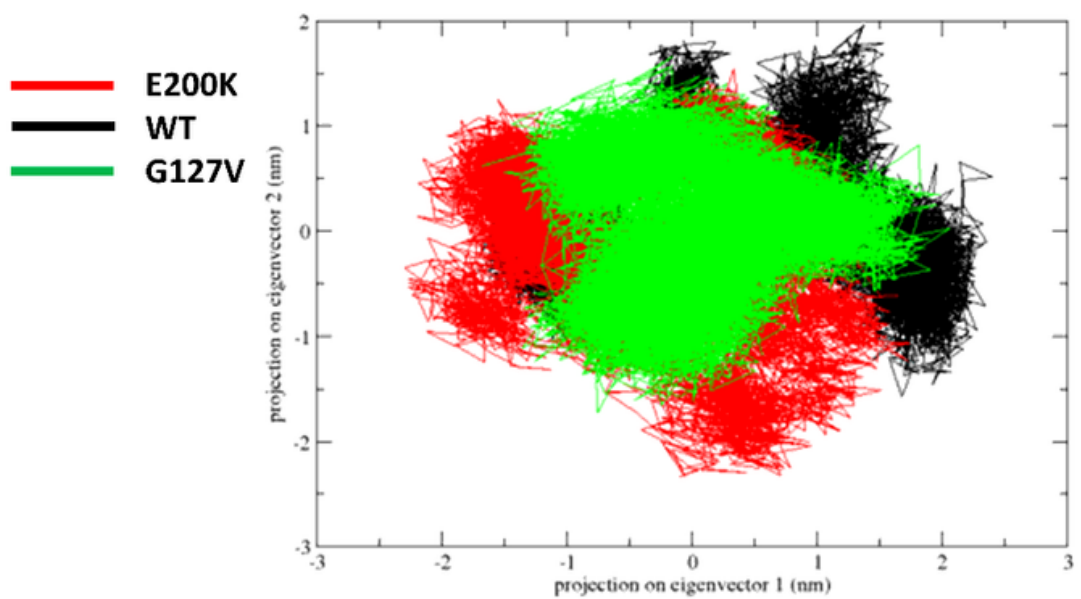

\section{Figure 6}

The principal component analysis for three models (WT, E200K, G127V). (A) The eigenvalues vs. eigenvector index plot. The first 50 eigenvectors have been taken into account. (B) Projection of the protein's motion in phase space along PC1 and PC2. 


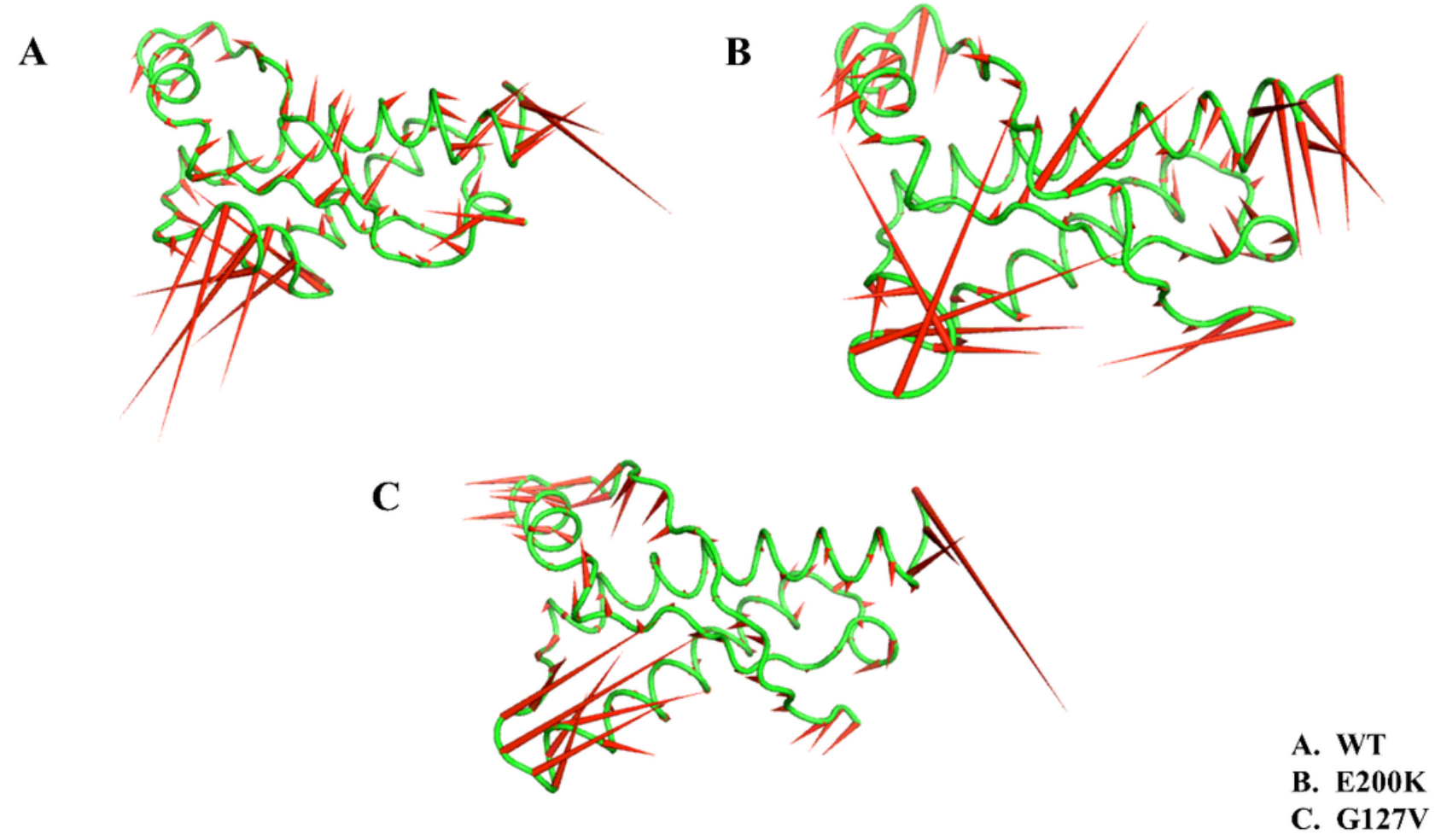

Figure 7

Porcupine plots of the PCA analysis depicting the movement and altitude of the C-alpha atoms throughout the 300 ns simulation in the WT (wild type), E200K, and G127V. 


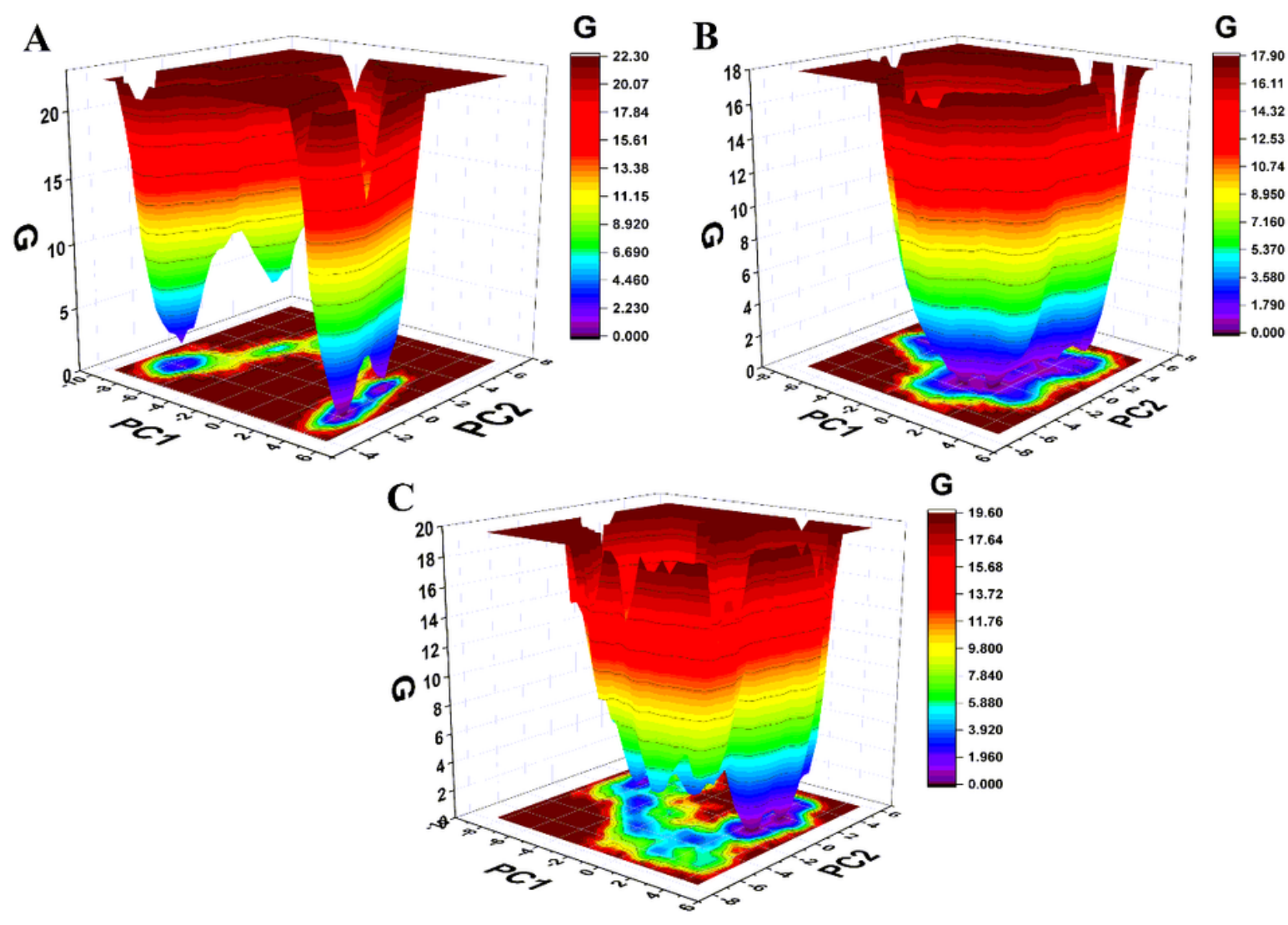

A. Wt

B. G127V

C. $\mathrm{E} 200 \mathrm{~K}$

Figure 8

The Gibbs free energy landscape plot of Prion protein in 3 models. (A) FEL for wild-type Prion protein (WT). (B) The mutant prion protein with E200K model and (C) The mutant Prion protein with G127V (resistant mutation) model. In the plot, the high energy state is indicated by the color red, and the low EFL is shown by blue. 